\title{
The Impact of Stripped Cores on the Frequency of Earth-size Planets in the Habitable Zone
}

\author{
Ilaria Pascucci ${ }^{1,2}$ (1), Gijs D. Mulders ${ }^{2,3}$ (1), and Eric Lopez ${ }^{4}$ \\ ${ }^{1}$ Lunar and Planetary Laboratory, The University of Arizona, Tucson, AZ 85721, USA; pascucci@lpl.arizona.edu \\ 2 Earths in Other Solar Systems Team, NASA Nexus for Exoplanet System Science, USA \\ ${ }^{3}$ Department of Geophysical Sciences, The University of Chicago, Chicago, IL, USA \\ ${ }^{4}$ NASA Goddard Space Flight Center, 8800 Greenbelt Road, Greenbelt, MD 20771, USA \\ Received 2019 May 7; revised 2019 July 18; accepted 2019 August 21; published 2019 September 19
}

\begin{abstract}
The frequency of Earth-size planets in the habitable zone (HZ) of Sun-like stars, hereafter $\eta_{\oplus}$, is a key parameter to evaluate the yield of nearby Earth analogs that can be detected and characterized by future missions. Yet, this value is poorly constrained as there are no reliable exoplanet candidates in the HZ of Sun-like stars in the Kepler field. Here, we show that extrapolations relying on the population of small $\left(<1.8 R_{\oplus}\right)$, short-period $(<25$ days $)$ planets bias $\eta_{\oplus}$ to large values. As the radius distribution at short orbital periods is strongly affected by atmospheric loss, we reevaluate $\eta_{\oplus}$ using exoplanets at larger separations. We find that $\eta_{\oplus}$ drops considerably, to values of only $\sim 5 \%-10 \%$. Observations of young $(<100 \mathrm{Myr})$ clusters can probe short-period sub-Neptunes that still retain most of their envelope mass. As such, they can be used to quantify the contamination of sub-Neptunes to the population of Kepler short-period small planets and aid in more reliable estimates of $\eta_{\oplus}$.
\end{abstract}

Key words: methods: data analysis - planets and satellites: detection - planets and satellites: terrestrial planets surveys

\section{Introduction}

The past decade has seen an exponential increase in the number of known exoplanets, mainly thanks to NASA's Kepler space telescope (e.g., Borucki et al. 2011; Borucki 2017). One of the most interesting and surprising results from this mission has been the discovery of a multitude of short-period planets (e.g., Fressin et al. 2013; Petigura et al. 2013), located much closer to their star than Mercury to the Sun. Follow-up observations of a subset of these planets (Johnson et al. 2017; Petigura et al. 2017) led to more precise stellar, hence planetary, radii and to the discovery of the so-called radius valley, a much lower frequency of planets with radii $\sim 1.8 R_{\oplus}$ rather than $\sim 1.3 R_{\oplus}$ or $\sim 2.4 R_{\oplus}$ (Fulton et al. 2017). Using the subset of exoplanet host stars with parameters homogeneously measured from asteroseismology, Van Eylen et al. (2018) confirmed the presence of the radius valley. Furthermore, they reported that the valley has a weak inverse dependence with orbital period as $\propto P^{-0.09}$, which has been recently confirmed by Martinez et al. (2019).

What is the origin of the radius valley? Owen \& Wu (2013) predicted early on that photoevaporation driven by high-energy stellar photons could herd planet radii into a bimodal distribution, closely matching that subsequently found by Fulton et al. (2017). This happens because photoevaporation is least efficient for planets that have twice the core radius, or an $\mathrm{H} / \mathrm{He}-\mathrm{rich}$ envelope that is just a few percent of the total mass: lighter or more massive envelopes are unstable and by evaporating efficiently end up populating one of the two peaks of the planet radius distribution (see Figure 6 in Owen \& Wu 2017; but also see Lopez \& Fortney 2013; Jin \& Mordasini 2018; Lopez \& Rice 2018). Alternatively, Ginzburg et al. (2018) suggested that the cooling luminosity of the planet itself drives atmospheric loss: light atmospheres, where the ratio between the heat capacity of the core and the envelope is $\leqslant 5 \%$, are mostly heated by the underlying rocky core and are rapidly removed while more massive atmospheres regulate their own cooling and can survive.

Importantly, both scenarios imply that the population of short-period $\left(<100\right.$ days), small $\left(<1.8 R_{\oplus}\right)$ planets is contaminated by sub-Neptunes that have lost a significant fraction of their envelope mass. Unlike Earth, these planets formed within few Myr in a gaseous circumstellar disk from which they accreted their envelope (e.g., Lee \& Chiang 2016). This conclusion is further corroborated by the expectation that a primordial rocky population, born after disk dispersal, should result in a larger planet mass, hence radius, with increasing semimajor axis (e.g., Lopez \& Rice 2018), which is opposite to the observed radius valley dependence with orbital period.

As the Kepler exoplanet detectability decreases rather steeply toward small planet radii and large orbital periods, and no true Earth analog ${ }^{5}$ has been discovered around Sun-like stars (e.g., Burke et al. 2015; Borucki 2017; Thompson et al. 2018), $\eta_{\oplus}$ cannot be directly measured. Values obtained from M or K dwarfs (e.g., Dressing \& Charbonneau 2015) likely provide an upper limit as small planets are more common around low-mass stars (e.g., see Mulders 2018a for a recent review on planet populations as a function of stellar properties). For Sun-like G-type stars, planets with either larger radii or much closer in to their stars have become crucial to estimate the frequency of Earth-size planets in the Habitable Zone (HZ), hereafter $\eta_{\oplus}$; see also Section 2. Lopez \& Rice (2018) pointed out that fitting separable power laws in planet radius and period will likely lead to an overestimate of $\eta_{\oplus}$ as the radius distribution will be dominated by short-period planets, many of which could be stripped cores, while the period distribution will be dominated by nonrocky sub-Neptunes.

Here, we begin to evaluate the impact of short-period planets on $\eta_{\oplus}$ in a systematic way. First, we explain our definition of

\footnotetext{
5 With Earth analog we mean Earth-size planet with an orbital period of 1 year.
} 
the $\mathrm{HZ}$ and review the methods and $\eta_{\oplus}$ estimates reported in the literature (Section 2). Next, we adopt the latest Kepler DR25 catalog (Thompson et al. 2018) with stellar properties from Gaia DR2 (Berger et al. 2018), in combination with the Exoplanet Population Observation Simulator epos ${ }^{6}$ (Mulders et al. 2018, hereafter M18), to evaluate the impact of shortperiod planets on estimates of $\eta_{\oplus}$. We show that $\eta_{\oplus}$ drops by factors of $\sim 4-8$ when extrapolations exclude short-period planets, many of which could be stripped cores (Section 2.1). As $\eta_{\oplus}$ directly impacts the yield of Earth analogs that can be detected by future missions like LUVOIR and HabEx (e.g., Stark et al. 2015), it is crucial to better constrain its value. A discussion of how this could be achieved is provided in Section 3.

\section{The Occurrence of Earth-size Planets in the $\mathbf{H Z}$}

One of the primary science goals of the Kepler mission was to measure the frequency of Earth-size and larger planets in the HZ of Sun-like stars (Borucki et al. 2003). As no true Earth analog has been detected, $\eta_{\oplus}$ estimates necessarily rely on assumptions based on the more abundant population of shortperiod and larger planets.

Numerous estimates of $\eta_{\oplus}$ are available in the literature and the ExoPAG Study Analysis Group 13 has recently summarized and tried to reconcile discrepancies among different studies. $^{7}$ To cancel out dependencies on the definition of the $\mathrm{HZ}$ and planet size range, the report focuses on comparing $\Gamma_{\oplus}=\left.\frac{\partial^{2} N(R, P)}{\partial \ln R \partial \ln P}\right|_{R=R_{\oplus}, P}=1 \mathrm{yr}$, i.e., $\eta_{\oplus}$ per $\log$ period and radius bin. Even with this definition, literature $\Gamma_{\oplus}$ span more than an order of magnitude in range, from 2\% (Foreman-Mackey et al. 2014) to $70 \%$ (Traub 2015). The report highlights that major differences are introduced by the use of different Kepler catalogs and completeness curves, with more recent ones giving systematically larger values, while different methods/ extrapolations introduce only a factor of two uncertainty. We will show, instead, that extrapolations are very sensitive to the exclusion of short-period planets (Section 2.1). Finally, the report provides power-law fits in period and radius for small $\left(<3.4 R_{\oplus}\right)$ planets based on the average of 12 community occurrence rate grids that include up to the DR2 4 Kepler data release. These fits imply $\Gamma_{\oplus}=38 \%$ or $\eta_{\oplus} \sim 20 \%$ when considering a conservative HZ (0.95-1.67 au) and habitable planets $\sim 0.8-1.4 R_{\oplus}$, very close to the $24 \%$ baseline value used to estimate the exoplanet yield for the $\mathrm{LUVOIR}^{8}$ and HabEX mission concept studies.

In M18 we used the latest Q1-Q17 DR2 5 Kepler catalog (Thompson et al. 2018) to present a new code, epos, which is based on a forward-modeling approach to constrain exoplanet populations. The code includes the most recent detection and vetting efficiency curves for the most reliable planet candidates (Robovetter score $\geqslant 0.9$ ). We fit two broken power laws, one in orbital period (for $2<P<400$ days) and one in planet radius (for $0.5<R<6 R_{\oplus}$ ), and demonstrated that they provide a good match to the bulk of the Kepler planet candidates (see the Appendix for a detailed description of the equations employed

\footnotetext{
6 Here we use the epos version 1.1.0 retrievable via https://github.com/ GijsMulders/epos.

7 https://exoplanets.nasa.gov/exep/exopag/sag/\#sag13

8 https://asd.gsfc.nasa.gov/luvoir/resources/docs/LUVOIR_Interim_ Report_Final.pdf

9 https://www.jpl.nasa.gov/habex/pdf/HabEx_Interim_Report.pdf
}

in epos). Integrating the posterior distribution in the $0.9<P<2.2 P_{\oplus}$ and $0.7<R<1.5 R_{\oplus}$ ranges, which is based on the Kopparapu et al. (2013) conservative HZ for the most common Sun-like stars in the Kepler sample, we found $\eta_{\oplus}=36_{-14}^{+14} \%$ and $\Gamma_{\oplus}=53_{-21}^{+20} \%$. These values agree with the baseline $\Gamma_{\oplus}=60 \%$ obtained by Burke et al. (2015) by fitting a single power law in period (for $50<P<300$ days) and broken power law in radius (for $0.75<R<2.5 R_{\oplus}$ ) on all Q1-Q16 Kepler planetary candidates, i.e., no reliability cut was applied. Hsu et al. (2019) recently estimated $\Gamma_{\oplus}$ from the DR25+Gaia DR2 catalog using a Bayesian framework and derived a median (50th percentile) value of $57 \%$ with $1 \sigma$ boundaries of $34 \%$ and $84 \%$, though they also did not include planets' reliability. Finally, Zink \& Hansen (2019) used the same DR25+Gaia DR2 catalog but adopted two independent broken power-law relations as in M18 and derived essentially the same $\eta_{\oplus}, 34 \%$, although with a much lower uncertainty of only $2 \%$ as they include several system's multiplicity parameters and priors to eliminate unphysical solutions.

\subsection{A Fourfold to Eightfold Drop in $\eta_{\oplus}$}

To evaluate the impact of short-period planets on $\eta_{\oplus}$ estimates, we adopt the same definition of $\mathrm{HZ}$ and habitable planets as in M18. We update epos to include Gaia-revised stellar radii for the Kepler sample (Berger et al. 2018) and recalculate detection efficiency contours for each individual star using KeplerPORTs (Burke \& Catanzarite 2017). After removing giants and subgiants as in Berger et al. (2018), we obtain a sample of 119,220 dwarfs with a median mass of $0.976 M_{\odot}$. We calculate the average survey detection efficiency for this sample as well as recompute vetting efficiency curves for the reliable (Robovetter score $\geqslant 0.9$ ) candidates in our sample following the approach described in M18.

Figure 1 shows our Kepler DR25+Gaia candidate list color coded by survey completeness. The gray rectangle delineates the HZ; no reliable detection is present in the region. Accepting all planet candidates, regardless of their Robovetter score, results in four detections, with two at the upper border of the box, while using pre-Gaia stellar parameters would further increase the number to 11; see also Figure 14 in Burke et al. (2015) for the same number of planetary candidates in the $\mathrm{HZ}$ from the Q1-Q16 Kepler catalog and pre-Gaia radii. ${ }^{10}$

We first run epos in its Monte Carlo mode with this new Kepler DR25+Gaia catalog, the updated completeness and vetting efficiencies, and over the same period $(2<P<400$ days) and planet radius $\left(0.5<R<6 R_{\oplus}\right)$ range as in M18. This first run is referred to as Model 1 . We find the same bestfit parameters as M18 within the quoted $1 \sigma$ confidence intervals; see Table 1. The posterior distributions (blue lines) and best-fit relations (black lines) in orbital period and planet radius for our Model 1 are shown in Figure 2. The same figure provides the occurrence rates calculated with the inverse detection efficiency method (red points with error bars). As already pointed out in M18, the low values for large orbital periods $(P \geqslant 30$ days $)$ and small planet radii $\left(R \leqslant 1.5 R_{\oplus}\right)$ are just due to the inclusion of bins where the completeness is low and Kepler has only partly detected planets (see Figure 1). To illustrate this point the green line in the lower panel of Figure 2

\footnotetext{
${ }^{10}$ Note that the five candidates at the completeness level of $0.01 \%$ were already marked as suspected false positives in the SAG13 report and excluded from occurrence rate calculations.
} 

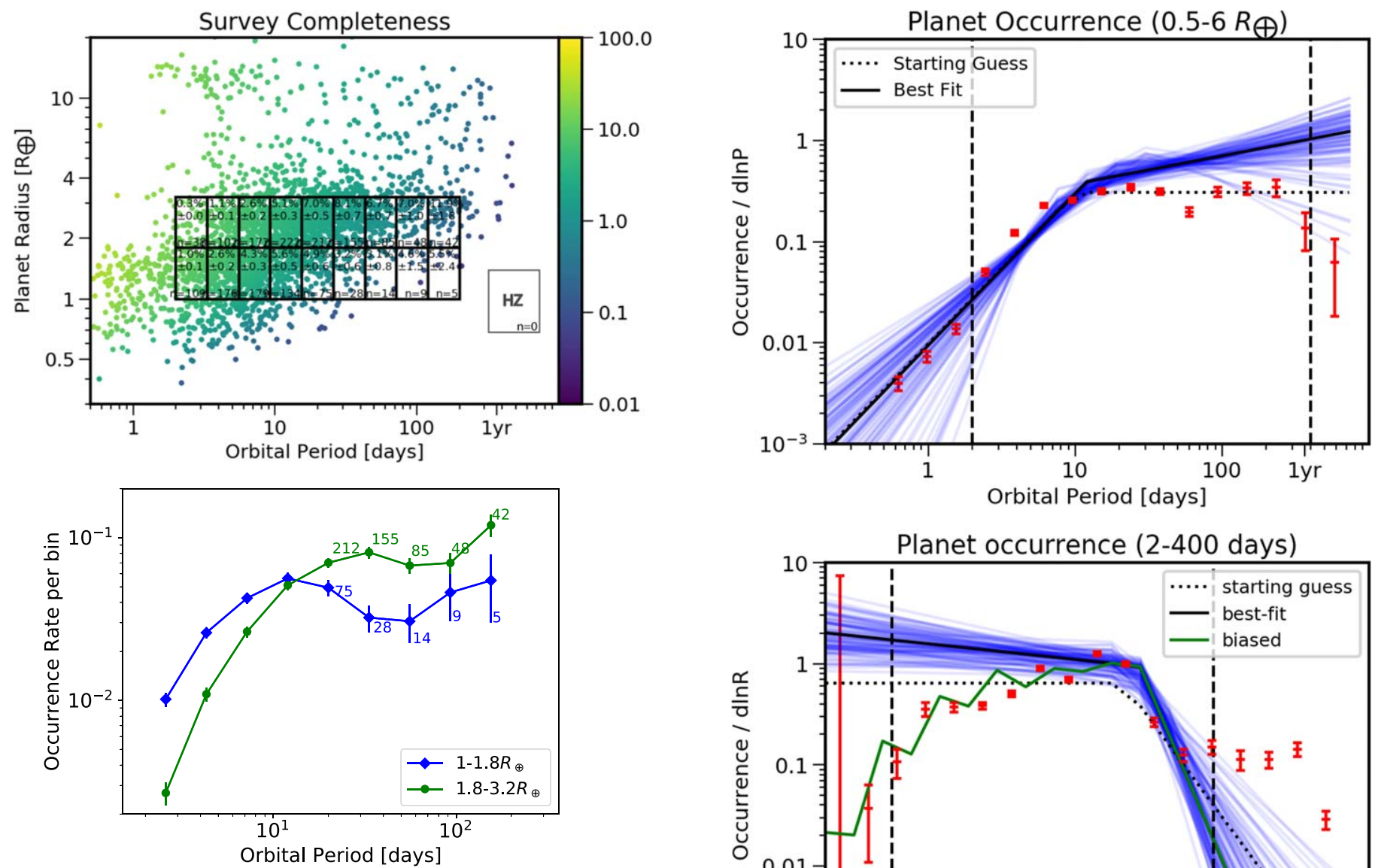

Figure 1. Upper panel: DR25+Gaia candidate list, color coded by survey completeness. The sample includes only dwarfs and planet candidates with a Robovetter score $\geqslant 0.9$. The gray rectangle delineates the HZ, no reliable planet candidate is detected inside the HZ. Occurrence rates using the inverse detection efficiency method are also provided for nine period and two radius bins (black rectangles). For clarity these values are also plotted in the lower panel with the number of planets per bin for the five bins at largest orbital periods.

Table 1

epos Best-fit Solutions with $1 \sigma$ Confidence Intervals

\begin{tabular}{lrrr}
\hline \hline Parameter & M18 & Model\#1 & Model\#4 \\
\hline$\eta$ & $4.9_{-1.2}^{+1.3}$ & $4.6_{-1.1}^{+1.0}$ & $2.7_{-0.3}^{+0.5}$ \\
$P_{\text {break }}$ (days) & $12_{-3}^{+5}$ & $11_{-3}^{+6}$ & $\ldots$ \\
$a_{P}$ & $1.5_{-0.3}^{+0.5}$ & $1.6_{-0.3}^{+0.6}$ & $\ldots$ \\
$b_{P}$ & $0.3_{-0.2}^{+0.1}$ & $0.3_{-0.2}^{+0.1}$ & $0.14_{-0.07}^{+0.07}$ \\
$R_{\text {break }}\left(R_{\oplus}\right)$ & $3.3_{-0.4}^{+0.3}$ & $3.4_{-0.3}^{+0.2}$ & $3.2_{-0.3}^{+0.3}$ \\
$a_{R}$ & $-0.5_{-0.2}^{+0.2}$ & $-0.3_{-0.2}^{+0.2}$ & $1.0_{-0.5}^{+0.5}$ \\
$b_{R}$ & $-6_{-3}^{+2}$ & $-7_{-2}^{+2}$ & $-6_{-2}^{+2}$ \\
\hline
\end{tabular}

Note. The equations used in the fit and an explanation for each of the parameters listed here are provided in the Appendix. Posterior distributions for Models 1 and 4 are shown in Figures 2 and 3, respectively.

gives the biased posterior, i.e., the posterior distribution assuming that no planets are detected below a completeness of $0.03 \%$. The good agreement between the red points and the green line demonstrates how the classic inverse detection efficiency method can underestimate true rates (see also Foreman-Mackey et al. 2014 and the Appendix for posteriors and occurrence rates over a restricted period and radius range with higher completeness). Note that the mismatch at $R>6 R_{\oplus}$

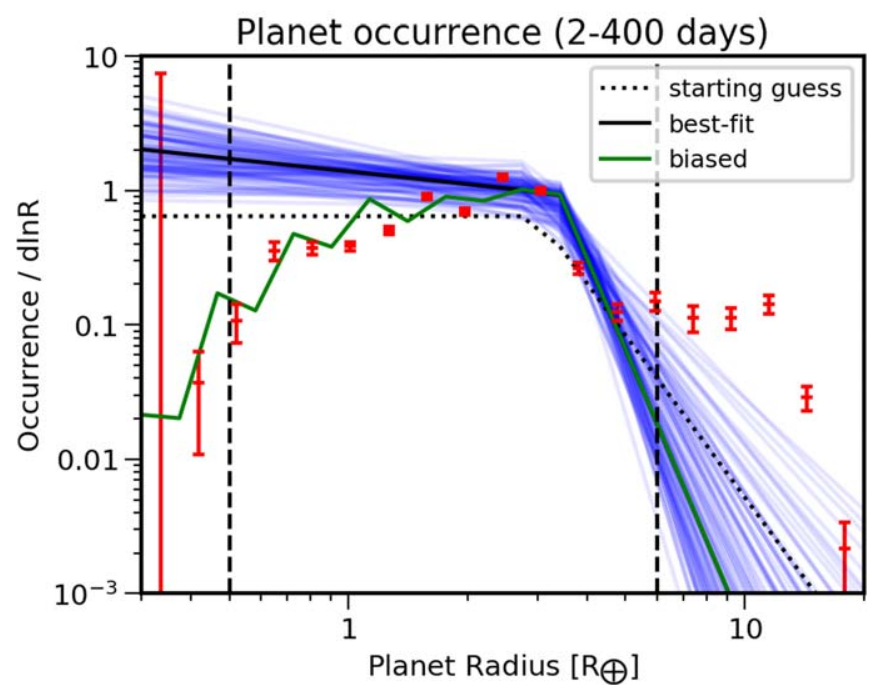

Figure 2. epos posterior orbital period (top) and planet radius distributions (bottom) for Model 1. Black vertical dashed lines indicate the range in planet period and radius that epos fits. Red points with error bars show the occurrence rates calculated with the inverse detection efficiency method. A biased version of the posterior planet radius distribution, assuming no planets below a completeness of $0.03 \%$, is shown in green. The good agreement between the green curve and the red points illustrates that occurrence rates, estimated with the inverse detection efficiency method, underestimate the true distribution in bins where the completeness is low and planets are only partially detected.

is inconsequential to this Letter since the fit and the $\eta_{\oplus}$ calculation ignore that part of the parameter space.

The key features of the best-fit relations shown in Figure 2 and relevant to this investigation are (i) a slight increase in the occurrence versus orbital period beyond $P_{\text {break }}$ and (ii) an increase in the occurrence of planets smaller than $R_{\text {break }}$. By integrating the posterior distribution in the $\mathrm{HZ}$ we find $\Gamma_{\oplus}=$ $60_{-25}^{+22} \%$ and $\eta_{\oplus}=41_{-17}^{+15} \%$; see also Table 2 , the same as those reported in M18. Expanding upon and corroborating M18, this test also shows that the Gaia-revised stellar radii have very little impact on this type of modeling, in spite of reducing by more than half the number of all candidates falling in the HZ; see also Zink \& Hansen (2019).

Next, we run epos over the same period range but only on the sample of small planets $\left(0.5<R<2 R_{\oplus}\right)$, i.e., we employ only a broken power law in period to fit the observed distribution (Model 2 in Table 2). This model returns similarly 
Table 2

epos Modeling Results

\begin{tabular}{llllrr}
\hline \hline $\begin{array}{l}\text { Model } \\
\#\end{array}$ & $\begin{array}{l}\text { Fitted } P \\
\text { days }\end{array}$ & $\begin{array}{l}\text { Fitted } R \\
R_{\oplus}\end{array}$ & Function & $\begin{array}{r}\Gamma_{\oplus} \\
\%\end{array}$ & $\begin{array}{r}\eta_{\oplus} \\
\%\end{array}$ \\
\hline 1 & $2-400$ & $0.5-6$ & 2 D broken & $59.6_{-25.4}^{+21.8}$ & $40.6_{-17.3}^{+14.9}$ \\
2 & $2-400$ & $0.5-2$ & $P$ broken & $78.7_{-39.2}^{+43.5}$ & $53.6_{-29.7}^{+29.7}$ \\
3 & $12-400$ & $0.5-6$ & $R$ broken & $17.0_{-5.6}^{+7.6}$ & $11.5_{-3.8}^{+5.2}$ \\
4 & $12-400$ & $1-6$ & $R$ broken & $16.0_{-5.5}^{+8.0}$ & $10.9_{-3.7}^{+5.5}$ \\
5 & $25-400$ & $0.5-6$ & $R$ broken & $8.6_{-5.1}^{+8.9}$ & $5.9_{-3.5}^{+6.0}$ \\
6 & $25-400$ & $1-6$ & $R$ broken & $8.0_{-5.4}^{+10.3}$ & $5.4_{-3.7}^{+7.0}$ \\
7 & $25-400$ & $1-2$ & $P$ and $R$ single & $7.8_{-3.8}^{+10.3}$ & $5.3_{-2.6}^{+7.0}$ \\
\hline
\end{tabular}

Note. "2D broken" stands for broken power law in period and radius while "P (R) broken" means that we have employed a broken power law in period (radius) and a single power law in radius (period); see the Appendix for the equations.

large $\Gamma_{\oplus}$ and $\eta_{\oplus}$ as Model 1 because the planet distribution increases toward small radii $\left(a_{R}=-1.25 \pm 0.05\right)$. Finally, we run a set of models where we exclude the population of shortperiod planets but fit a large range of planet radii with a broken power law. The minimum period of 12 days (Models 3 and 4) is chosen to exclude the known orbital period break for subNeptunes (Howard et al. 2012), while for periods $>25$ days (Models 5, 6, and 7) theoretical models predict minimal photoevaporation (e.g., Owen \& Wu 2017), hence negligible contamination of stripped cores. We find that $\Gamma_{\oplus}$ and $\eta_{\oplus}$ drop by factors of $\sim 4-8$ when excluding the population of shortperiod planets and that the results are robust against the lower planet radius boundary that is adopted. Note that Model 7, single power laws for small planets with minimal photoevaporation, essentially provides the same $\eta_{\oplus}$ estimates as Model 6 where the inclusion of large planets is modeled via a broken power-law relation in planet radius.

To clarify why there is such a difference in the $\eta_{\oplus}$ estimates, we provide the epos best-fit values and $1 \sigma$ confidence intervals for Model 4 in Table 1 and the posterior orbital period and planet radius distributions in Figure 3. The planet distribution is still slightly increasing toward larger orbital periods (parameter $b_{P}$ ) but steeply drops toward small planet radii (parameter $a_{R}$ ). It is the difference in the best-fit powerlaw index for small planets $\left(<3 R_{\oplus}\right)$ that leads to a fourfold drop in $\eta_{\oplus}$ between Models 1 and 4 .

The lower panel of Figure 1 further clarifies why excluding short-period planets results in smaller $\eta_{\oplus}$. It shows that the occurrence of small (1-1.8 $\left.R_{\oplus}\right)$ planets, calculated by applying the inverse detection efficiency method over bins with relatively high completeness $>0.01 \%$, drops by almost a factor of $\sim 2$ from the $\sim 10$ day bin to the $\sim 30$ day bin. In contrast, the occurrence of large $\left(1.8-3.2 R_{\oplus}\right)$ planets increases by $\sim 50 \%$ over the same bins and continues to increase out to 200 days. Note that the small planets' $\sim 30$ day bin has an even higher survey completeness that the bin at 120 days for the $1.8-3.2 R_{\oplus}$ planets and does not fall below $0.01 \%$ out to 200 days. Hence, the drop beyond $\sim 10$ days in the planet occurrence of $1-1.8 R_{\oplus}$ planets is robust. This drop further indicates that the occurrence of small, short-period planets is not representative for the one at longer orbits, and such planets should not be included to infer $\eta_{\oplus}$. Interestingly, fitting the occurrence of $1-1.8 R_{\oplus}$ planets beyond 12 days with a single power law gives an index of 0.13 , the same as $b_{p}$ for Model 4 , and an occurrence of $10 \%$ when integrated over the $\mathrm{HZ}$ period of $0.9-2.2 P_{\oplus}$, similar to the low
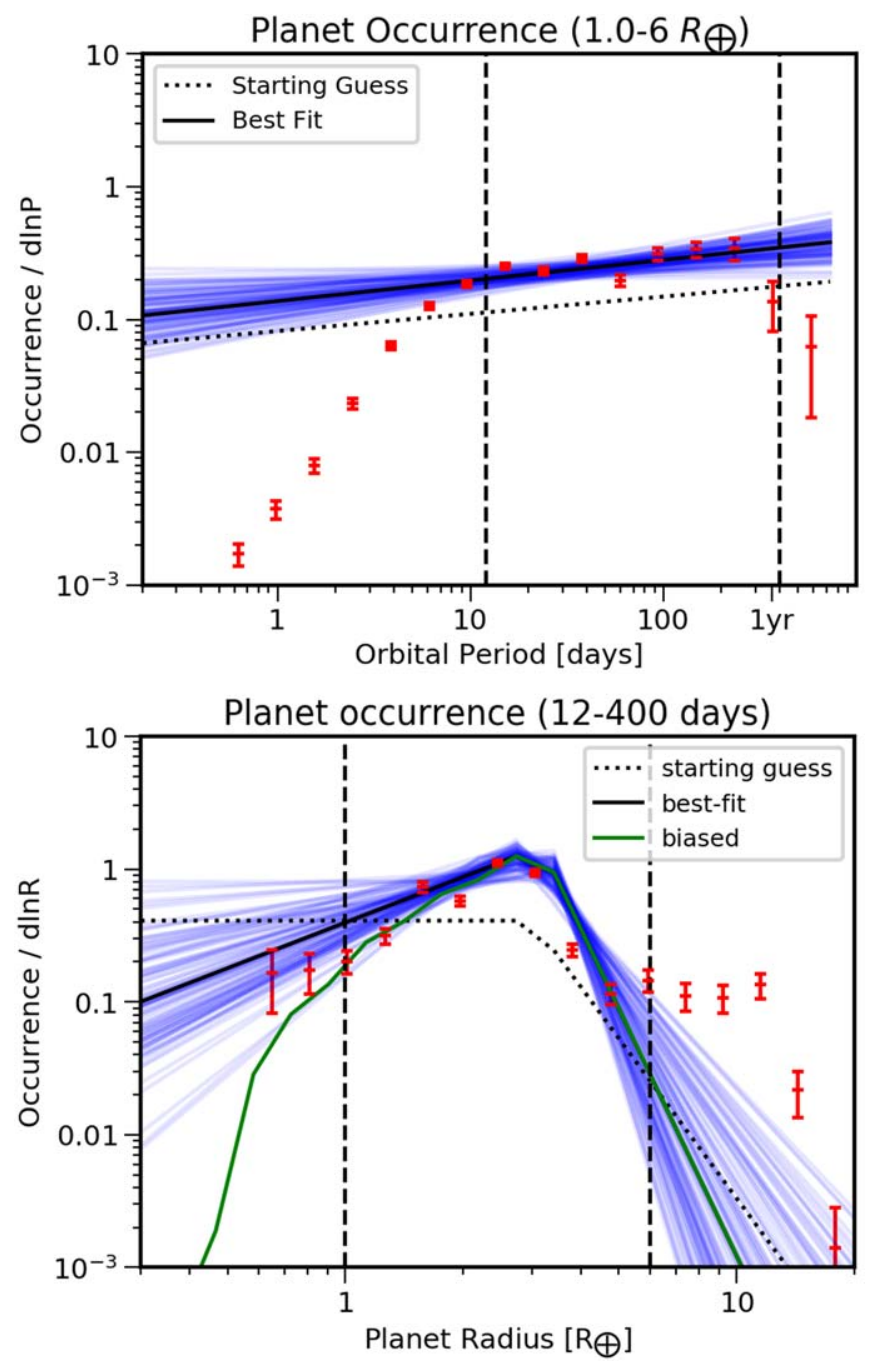

Figure 3. epos posterior orbital period distribution (top) and planet radius distribution (bottom) for Model 4. Symbols as in Figure 2. Note that these occurrence rates (red points with error bars) are calculated for larger planets than in Figure 2; hence, they underestimate the true distribution less.

$\eta_{\oplus}$ values calculated by epos when excluding the population of small, short-period planets.

\section{Discussion and Outlook}

When considering the sample of most reliable Kepler candidates (Robovetter score $\geqslant 0.9$ ), there are no exoplanets detected in the HZ of Sun-like stars. As such, extrapolations are necessary to estimate $\eta_{\oplus}$. Here, we have shown that extrapolations relying on the population of small $\left(<1.8 R_{\oplus}\right)$, short-period ( $<25$ days) planets bias $\eta_{\oplus}$ to large values, mainly because the inferred distribution versus planet radius increases toward Earth-sized planets. Excluding this population leads to a fourfold to eightfold drop in $\eta_{\oplus}$. The existence of the radius valley (Fulton et al. 2017), combined with its orbital period dependence (Van Eylen et al. 2018; Martinez et al. 2019), provides strong support that the population of small, shortperiod planets is contaminated by stripped cores. Therefore, the occurrence of small, short-period planets is not representative of that of planets farther away from the star and should not be used to infer the frequency of rocky planets in the HZ. Supporting this statement we have shown that, in the region 
with high survey completeness and for the most reliable Kepler candidates, the population of small $\left(<1.8 R_{\oplus}\right)$ planets drops beyond 10 days. How can we then obtain more reliable estimates of $\eta_{\oplus}$ ?

Independent transit or radial velocity detections of small, long-period ( $>100$ days) Kepler candidates would provide the most robust approach to measure the frequency of rocky planets close to the HZ. Such follow-ups would identify the true planets, thus eliminating the use of candidates with a chosen reliability cut, and, being at relatively large orbital periods, reduce the uncertainty when extrapolating into the $\mathrm{HZ}$. While there are a few ongoing efforts (e.g., Burke et al. 2019), the faintness of the Kepler stars, combined with the large orbital period and transit duration of these candidates, makes it unlikely that all of them can be independently confirmed. Statistical validation, which includes ancillary observational evidence, has been also pursued (e.g., Torres et al. 2017), but it cannot be extended to long-period, low signal-to-noise planets (Mullally et al. 2018; Burke et al. 2019).

Another approach is to quantify the contamination of subNeptunes with significantly reduced envelope mass to the population of small, short-period planets. Understanding whether photoevaporation or core-powered mass loss dominate would be an important first step. As core-powered mass loss correlates with the bolometric luminosity of the star, while photoevaporation is driven by high-energy stellar photons, characterizing the radius valley for stars of different spectral types could help distinguishing between these two mechanisms (e.g., Ginzburg et al. 2018). In addition, quantitative comparisons between both models and the Kepler data, carried out in the same uniform way, would be extremely valuable to test them. Such comparisons could reveal analytic relations for the period-radius distribution under the influence of atmospheric loss that could be included in epos and used to refine $\eta_{\oplus}$ estimates. Alternatively, observations of young ( $\leqslant 100 \mathrm{Myr})$ clusters with TESS could measure the occurrence of primordial short-period, large planets $\left(1.8-3.2 R_{\oplus}\right)$. Subtracting from this population the corresponding old planet population would give the frequency of sub-Neptunes whose atmosphere has been significantly stripped away from photoevaporation or planet's cooling. Finally, removing this population from the Kepler short-period small $\left(1-1.8 R_{\oplus}\right)$ planets would unveil the occurrence of rocky planets that formed like Earth.

This material is based upon work supported by the National Aeronautics and Space Administration under Agreement No. NNX15AD94G for the program Earths in Other Solar Systems. The results reported herein benefited from collaborations and/ or information exchange within NASA's Nexus for Exoplanet System Science (NExSS) research coordination network sponsored by NASA's Science Mission Directorate.

Facility: Kepler.

Software: astropy (Astropy Collaboration et al. 2013), emcee (Foreman-Mackey et al. 2013), epos (Mulders 2018b), KeplerPORTs (Burke \& Catanzarite 2017).

\section{Appendix epos Parametric Fit}

Here, we briefly summarize the key equations used in epos to fit the observed Kepler exoplanet population. We direct the reader to M18 for a complete description of the code and examples on how to use it. ${ }^{11}$ The planet occurrence rate distribution is described with separable functions in period $P$ and planet radius $R$ :

$$
\frac{d N}{d \log P d \log R}=A f(P) f(R),
$$

where $A$ is a normalization factor and the integral of the function over the simulated planet period and radius range equals the number of planets per star $(\eta)$. In M18, as well as in Models 1 and 2 of this Letter, the planet orbital period distribution is described by a broken power law:

$$
f(P)=\left\{\begin{array}{ll}
\left(\frac{P}{P_{\mathrm{br}}}\right)^{a_{P}} & \text { if } P<P_{\mathrm{br}} \\
\left(\frac{P}{P_{\mathrm{br}}}\right)^{b_{P}} & \text { otherwise }
\end{array},\right.
$$

where the break in orbital period at $\sim 10$ days for sub-Neptunes was first recognized by Youdin (2011) and Howard et al. (2012) and likely reflects the inner edge of protoplanetary disks (Mulders et al. 2015). When fitting a large range of planet radii the radius distribution also follows a broken power law:

$$
f(R)= \begin{cases}\left(\frac{R}{R_{\mathrm{br}}}\right)^{a_{R}} & \text { if } R<R_{\mathrm{br}} \\ \left(\frac{R}{R_{\mathrm{br}}}\right)^{b_{R}} & \text { otherwise }\end{cases}
$$

reflecting early findings of a departure from a single power law at $\sim 2 R_{\oplus}$ (Petigura et al. 2013). This type of broken power law in radius is used in Models 3-6 in this Letter. epos generates a synthetic planet population via a Monte Carlo approach by random draws from the distributions outlined above. Uncertainties on the best-fit parameters are obtained via a Markov Chain Monte Carlo simulation using emcee (ForemanMackey et al. 2013). For each simulation presented in this study we used 200 walkers for 5000 Monte Carlo iterations and a 1000-step burn-in.

Figure 4 shows the epos posterior orbital period and planet radius distributions for a model analog to Model 1 , but with the fit restricted in planet period (2-200 days) and radius (1-6 $\left.R_{\oplus}\right)$. This new model results in the same best-fit solutions as Model 1 , which is why it is not included in the main text, but illustrates how the inverse detection efficiency method (red points with error bars) can underestimate the true occurrence in a bin that includes regions with low survey completeness and planets detected only in part of the bin (compare the red points in Figure 2 and Figure 4). The limitations of the inverse detection efficiency method were also pointed out in ForemanMackey et al. (2014), which is why the forward-modeling approach in epos is preferable, especially in regions with few planet detections.

\section{ORCID iDs}

Ilaria Pascucci (1D https://orcid.org/0000-0001-7962-1683 Gijs D. Mulders (10 https://orcid.org/0000-0002-1078-9493

\footnotetext{
11 https://github.com/GijsMulders/epos
} 

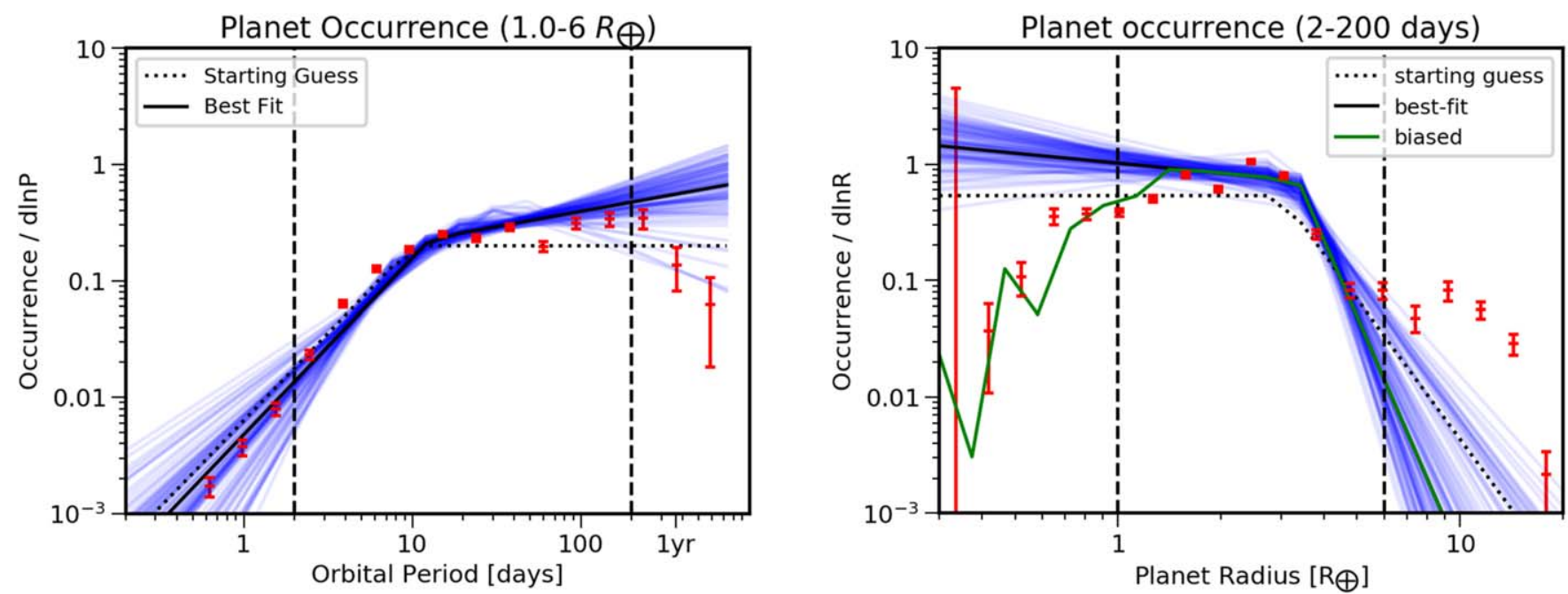

Figure 4. epos posterior orbital period distribution (left) and planet radius distribution (right) for a 2D broken power law as for Model 1 but with the fit restricted in planet period (2-200 days) and radius (1-6 $\left.R_{\oplus}\right)$. Note that the occurrence rates obtained with the inverse detection method (red points with error bars) are closer to the best fit than in Figure 2 as this period and radius range includes fewer bins with low completeness and only partly detected planets.

\section{References}

Astropy Collaboration, Robitaille, T. P., Tollerud, E. J., et al. 2013, A\&A, $558, \mathrm{~A} 33$

Berger, T. A., Huber, D., Gaidos, E., \& van Saders, J. L. 2018, ApJ, 866, 99 Borucki, W. J. 2017, PAPhS, 161, 38

Borucki, W. J., Koch, D., Basri, G., et al. 2003, ESASP, 539, 69

Borucki, W. J., Koch, D. G., Basri, G., et al. 2011, ApJ, 728, 117

Burke, Ch. J., Christiansen, J. L., Mullally, F., et al. 2015, ApJ, 809, 8

Burke, C. J., \& Catanzarite, J. 2017, Planet Detection Metrics: Per-Target Detection Contours for Data Release, 25, (KSCI-19111-002)

Burke, C. J., Mullally, F., Thompson, S. E., Coughlin, J. L., \& Rowe, J. F. 2019, AJ, 157, 143

Dressing, C. D., \& Charbonneau, D. 2015, ApJ, 807, 45

Foreman-Mackey, D., Hogg, D. W., Lang, D., \& Goodman, J. 2013, PASP, 125,306

Foreman-Mackey, D., Hogg, D. W., \& Morton, T. D. 2014, ApJ, 795, 64

Fressin, F., Torres, G., Charbonneau, D., et al. 2013, ApJ, 766, 81

Fulton, B. J., Petigura, E. A., Howard, A. W., et al. 2017, AJ, 154, 109

Ginzburg, S., Schlichting, H. E., \& Sari, R. 2018, MNRAS, 476, 759

Howard, A. W., Marcy, G. W., Bryson, S., et al. 2012, ApJS, 201, 15

Hsu, D. C., Ford, E. B., Ragozzine, D., \& Ashby, K. 2019, AJ, 158, 109

Jin, S., \& Mordasini, C. 2018, ApJ, 853, 163
Johnson, J. A., Petigura, E. A., Fulton, B., et al. 2017, AJ, 154, 108 Kopparapu, R. K., Ramirez, R., Kasting, J., et al. 2013, ApJ, 765, 131 Lee, E. J., \& Chiang, E. 2016, ApJ, 817, 90

Lopez, E. D., \& Fortney, J. J. 2013, ApJ, 776, 2

Lopez, E. D., \& Rice, K. 2018, MNRAS, 479, 5303

Martinez, C. F., Cunha, K., Ghezzi, L., \& Smith, V. V. 2019, ApJ, 875, 29 Mulders, G. 2018b, AJ, 156, 24

Mulders, G. D. 2018a, Handbook of Exoplanets (Berlin: Springer)

Mulders, G. D., Pascucci, I., \& Apai, D. 2015, ApJ, 798, 112

Mulders, G. D., Pascucci, I., Apai, D., \& Ciesla, F. J. 2018, AJ, 156, 24, (M18) Mullally, F., Thompson, S. E., Coughlin, J. L., Burke, C. J., \& Rowe, J. F. 2018, AJ, 155, 210

Owen, J. E., \& Wu, Y. 2013, ApJ, 775, 105

Owen, J. E., \& Wu, Y. 2017, ApJ, 847, 29

Petigura, E. A., Howard, A. W., Marcy, G., et al. 2017, AJ, 154, 107

Petigura, E. A., Marcy, G. W., \& Howard, A. W. 2013, ApJ, 770, 69

Stark, Ch. C., Roberge, A., Mandell, A., et al. 2015, ApJ, 808, 149

Thompson, S. E., Coughlin, J. L., Hoffman, K., et al. 2018, ApJS, 235, 38

Torres, G., Kane, S. R., Rowe, J. F., et al. 2017, AJ, 154, 264

Traub, W. A. 2015, IJAsB, 14, 359

Van Eylen, V., Agentoft, C., Lundkvist, M. S., et al. 2018, MNRAS, 479, 4786

Youdin, A. N. 2011, ApJ, 742, 38

Zink, J. K., \& Hansen, B. M. S. 2019, MNRAS, 487, 246 